\title{
Identification of Flavonoids as Putative ROS-1 Kinase Inhibitors Using Pharmacophore Modeling for NSCLC Therapeutics
}

\author{
Shraddha Parate ${ }^{1}\left({ }^{1}\right.$, Vikas Kumar $^{2}{ }^{\circledR}$, Jong Chan Hong ${ }^{1, *}$ and Keun Woo Lee ${ }^{2, *}$ \\ 1 Division of Applied Life Science, Plant Molecular Biology and Biotechnology Research Center (PMBBRC), \\ Gyeongsang National University (GNU), 501 Jinju-daero, Jinju 52828, Korea; parateshraddha@gmail.com \\ 2 Division of Life Sciences, Department of Bio \& Medical Big Data (BK21 Four Program), Research Institute of \\ Natural Science (RINS), Gyeongsang National University (GNU), 501 Jinju-daero, Jinju 52828, Korea; \\ vikaspathania777@gmail.com \\ * Correspondence: jchong@gnu.ac.kr (J.C.H.); kwlee@gnu.ac.kr (K.W.L.)
}

check for updates

Citation: Parate, S.; Kumar, V.; Hong J.C.; Lee, K.W. Identification of Flavonoids as Putative ROS-1 Kinase Inhibitors Using Pharmacophore Modeling for NSCLC Therapeutics. Molecules 2021, 26, 2114. https:// doi.org/10.3390/molecules26082114

Academic Editor: Julio Caballero

Received: 4 March 2021

Accepted: 5 April 2021

Published: 7 April 2021

Publisher's Note: MDPI stays neutral with regard to jurisdictional claims in published maps and institutional affiliations.

Copyright: (c) 2021 by the authors. Licensee MDPI, Basel, Switzerland. This article is an open access article distributed under the terms and conditions of the Creative Commons Attribution (CC BY) license (https:// creativecommons.org/licenses/by/ $4.0 /)$.

\begin{abstract}
Non-small cell lung cancer (NSCLC) is a lethal non-immunogenic malignancy and protooncogene ROS-1 tyrosine kinase is one of its clinically relevant oncogenic markers. The ROS-1 inhibitor, crizotinib, demonstrated resistance due to the Gly2032Arg mutation. To curtail this resistance, researchers developed lorlatinib against the mutated kinase. In the present study, a receptor-ligand pharmacophore model exploiting the key features of lorlatinib binding with ROS-1 was exploited to identify inhibitors against the wild-type (WT) and the mutant (MT) kinase domain. The developed model was utilized to virtually screen the TimTec flavonoids database and the retrieved drug-like hits were subjected for docking with the WT and MT ROS-1 kinase. A total of 10 flavonoids displayed higher docking scores than lorlatinib. Subsequent molecular dynamics simulations of the acquired flavonoids with WT and MT ROS-1 revealed no steric clashes with the Arg2032 (MT ROS-1). The binding free energy calculations computed via molecular mechanics/Poisson-Boltzmann surface area (MM/PBSA) demonstrated one flavonoid (Hit) with better energy than lorlatinib in binding with WT and MT ROS-1. The Hit compound was observed to bind in the ROS-1 selectivity pocket comprised of residues from the $\beta-3$ sheet and DFG-motif. The identified Hit from this investigation could act as a potent WT and MT ROS-1 inhibitor.
\end{abstract}

Keywords: ROS-1 kinase; drug resistance; NSCLC; flavonoids; structure-based pharmacophore; virtual screening; molecular docking; molecular dynamics simulations; MM/PBSA

\section{Introduction}

Lung cancer remains one of the fatal causes of cancer related malignancies in the world with non-small cell lung cancer (NSCLC) accounting for $85 \%$ of cases [1]. NSCLC encompasses numerous subtypes, including lung adenocarcinoma (LUAD) and lung squamous cell carcinoma (LUSC) and despite advances in diagnosis, 70\% of patients present with metastatic lung cancer disease when surgery is not an option [2]. Receptor tyrosine kinases (RTKs) have emerged as critical players of cellular communication function and network. Activating fusions and rearrangement of the proto-oncogene ROS-1 (c-ros oncogene 1) have been reported in $1-2 \%$ of NSCLC cases as potent oncogenic drivers, often found in non-smokers and patients of younger age [3]. Furthermore, oncogenic ROS-1 leads to the activation of several components of the PI3K/AKT/mTOR signaling pathway [4]. Numerous ROS-1 fusion proteins have been identified and reported in literature including SDC4, TMEM106B-ROS-1, SLC34A2-ROS-1, TPM3, CD74-ROS-1, LRIG3, EZR, CLTC, TPD52L1-ROS-1, CCDC6 and FIG-ROS-1 [5-8].

Many researchers are pouring tremendous efforts into developing effective and efficient ROS-1 inhibitors [9]. The ROS-1 gene has 49\% sequence homology in the kinase domain and 77\% identity at the ATP-binding site with anaplastic lymphoma kinase (ALK), thereby forming the basis for the utilization of ALK inhibitors for ROS-1 rearranged NSCLC 
patients [10]. To date, most of the ROS-1 inhibitors with remarkable in vitro inhibitory activities are the repurposing of first- and second-generation inhibitors of ALK, including alectinib, ceritinib, foretinib and crizotinib [11-14]. The first-generation ALK inhibitor, crizotinib (Xalkori ${ }^{\circledR}$, Pfizer), was granted FDA approval in 2016 for the treatment of ROS-1 rearranged NSCLC [9]. However, the insurgence of Gly2032Arg mutation in the ROS-1 kinase domain conferred resistance to crizotinib treatment in ROS-1 fusion-positive NSCLC patients [15]. The Gly2032 residue at the solvent front in the rigid phosphate-binding loop (P-loop) of the kinase domain interacts with the pyrazole group of crizotinib via van der Waals forces. The mutated Arg2032 residue partially occupies the pyrazole binding space, thereby interfering with crizotinib binding and leading to resistance and steric clashes with its piperidine ring [16]. To overcome this, a third-generation ROS-1/ALK inhibitor, lorlatinib (PF 06463922, Pfizer), was designed lacking the piperidine ring, which was replaced by a pyridine ring for preventing the Arg2032-induced steric clashes [16]. Additional structural changes to handle drug resistance were also made in lorlatinib leading to better therapeutic efficacy than crizotinib. Another tyrosine kinase inhibitor, Entrectinib (ROZLYTREK, Genentech Inc.), was approved by FDA in 2019 for ROS-1 altered lung cancers [17]. The multi-kinase inhibitor, Cabozantinib, also demonstrated effective clinical efficacy in ROS-1 positive NSCLC patients [18]. Despite demonstrating efficient activity against ROS- 1 cancers, the aforementioned drugs showed limited efficacy against Gly2032Arg ROS-1 mutants [19]. Therefore, there is an emergent need to develop efficient new inhibitors, selective for ROS-1 that would overcome Gly2032Arg-mediated resistance.

Phytochemicals are plant-derived natural compounds used to treat several ailments, including cancer, and numerous reports have demonstrated their influence on tumor proliferation and metastasis via in vitro and in vivo studies [20]. Moreover, natural products exhibit greater potential druggable pharmacophores when compared to synthetic compounds characterized by their wide availability, cost-effectiveness and high tolerance [20]. These polyphenols comprise flavonoids, stilbenes, lignans and phenolic acids among which flavonoids constitute approximately two-thirds of plant polyphenols in the human diet [21]. Natural flavonoids categories, including flavonols, anthocyanins, isoflavones and flavones, exert beneficial effects on human health and dietary intake and represent a novel therapeutic template for the design of cancer inhibitors [20]. Furthermore, flavonoids scavenge the free radicals involved in DNA damage and tumor progression [22]. The protective ability of some of the flavonoids is well-documented in the literature with quercetin and naringin as examples [22]. A study published recently reported on Trifolium flavonoids in overcoming gefitinib resistance of NSCLC by downregulating the STAT3 and ERK pathways [23]. The anti-cancer effects of flavonoids on lung cancer cell lines were also reported via inhibition of the Wnt/ $\beta$-catenin signaling pathway [24]. Flavonoids have also exhibited cytotoxic potential against lung cancer cell lines in several studies by arresting the progression of the cell cycle and inducing cancer cell apoptosis [25-27]. More importantly, the structural backbone of flavonoids contributes to the killing of resistant cancer cells [28].

On the basis of the above-mentioned perspectives, we sought to identify natural product flavonoids as potential therapeutics targeted to ROS-1 tyrosine kinase for the NSCLC treatment. We have carried out an in silico study considering the development of a receptor-based pharmacophore model for virtual screening by adopting the ROS-1lorlatinib interaction as a template structure. The developed pharmacophore model has been utilized to virtually screen flavonoids from the TimTec database, and subsequently, the acquired flavonoids were filtered for their drug-likeness via Lipinski's, Veber's and ADMET filters. The binding interaction of obtained drug-like flavonoids was investigated with the ROS-1 kinase domain pocket residues. Additionally, the flavonoids obtained from docking analysis with ROS-1 kinase domain were subjected to extensive molecular dynamics (MD) simulations to check their stability over a period of $50 \mathrm{~ns}$. The flavonoids demonstrating binding interactions with key residues of the ROS-1 kinase domain similar to that observed in the ROS-1-lorlatinib crystallographic structure were additionally docked with the constructed Gly2032Arg ROS-1 mutant structure. The flavonoids are 
exhibiting interactions with Gly2032 and Arg2032 in wild type (WT) and mutant (MT) ROS-1, respectively, which were identified as molecules with potent scaffolds that could inhibit ROS-1. Furthermore, the binding free energy (BFE) calculations executed through MM/PBSA (molecular mechanics/Poisson-Boltzmann surface area) displayed effective binding affinity of flavonoids with WT and MT ROS-1 kinase domain.

\section{Results}

\subsection{Receptor-Ligand Pharmacophore Model}

A receptor-ligand (structure-based) pharmacophore model was generated from the crystallographic structure of ROS-1 tyrosine kinase complexed with its selective inhibitor, lorlatinib (PDB ID: 4UXL) [16]. The ROS-1 selective inhibitor, lorlatinib exhibited improved activity $\left(\mathrm{IC}_{50}=0.6 \mathrm{nM}\right)$ against ROS-1 compared to dual ALK/ROS-1 inhibitor, crizotinib $\left(\mathrm{IC}_{50}=253.7 \mathrm{nM}\right)$ and also demonstrated in vivo activity in patients expressing Gly2032Arg mutation [16]. The key pharmacophoric features of lorlatinib binding with ROS-1 were explored, resulting in the generation of only one pharmacophore hypothesis with one hydrogen bond donor (HBD), one hydrogen bond acceptor (HBA), and two hydrophobic (Hy) features as essential features (Table 1, Figure 1). The Pharmacophore_01 with a selectivity score of 7.0747 indicated that lorlatinib interacts with Glu2027 and Met2029 via $\mathrm{HBD}$ and HBA features, respectively forming hydrogen bond interactions, while hydrophobic interactions with residues Val1959 (alkyl), Ala1978 ( $\pi$-alkyl), Lys1980 (alkyl), Leu2026 (alkyl) and Leu2086 ( $\pi$-sigma) are observed via Hy pharmacophoric feature. Lorlatinib additionally formed several van der Waals interactions with residues including Leu2010, Gly2032, Asp2033, Arg2083 and Asp2102. A study by Pathak et al. reported the structure-based pharmacophore model where they screened two commercial databasesMaybridge and Chembridge. The authors were able to successfully acquire five hits with the aforementioned pharmacophoric features, capable of inhibiting the WT and MT ROS-1 proteins [29]. Therefore, the Pharmacophore_01 model was taken forward for screening the database of flavonoids.

Table 1. Structure-based pharmacophore model summary with its generated features.

\begin{tabular}{cccc}
\hline Pharmacophore Models & Number of Features & Feature Set & Selectivity Score \\
\hline Pharmacophore_01 & 4 & ADHH & 7.0747 \\
\hline${ }^{*}$ A: hydrogen bond acceptor (HBA); D: hydrogen bond donor (HBD); H: hydrophobic (Hy).
\end{tabular}
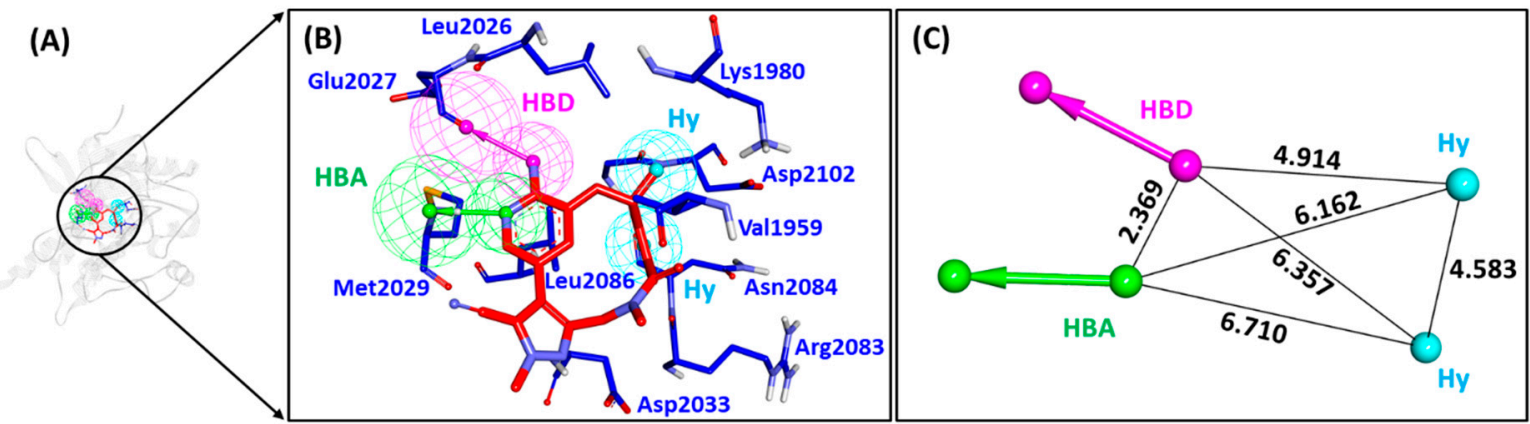

Figure 1. Receptor-ligand pharmacophore model- Pharmacophore_01. (A) Pharmacophore model generated at the catalytic site of c-ros oncogene1 (ROS-1) complexed with its co-crystallized ligand, lorlatinib. (B) ROS-1 selective inhibitor, lorlatinib mapping with essential residues of the ROS-1 active site via key pharmacophoric features- HBD, HBA and Hy. (C) Interfeature distance between the generated features of the pharmacophore. HBD (hydrogen bond donor); HBA (hydrogen bond acceptor); Hy (hydrophobic). 


\subsection{Güner-Henry Validation of the Receptor-Ligand Pharmacophore Model}

The Pharmacophore_01 model generated above was validated via Güner-Henry method, also referred to as decoy set validation, used for measuring the overall suitability of the model in picking active compounds from decoys. An external database (D) of 78 molecules with 20 actives (A) ROS-1 inhibitors was prepared with the remaining molecules as decoy molecules. The Pharmacophore_01 model was able to retrieve 25 hits (Ht) with 20 active compounds (Ha), resulting in the goodness of fit (GF) score of 0.77 . The GF score was observed to be near the ideal model range value [30], thereby confirming the selectivity of our model in picking active compounds from decoys (Table 2).

Table 2. Decoy set validation of Pharmacophore_01 from an external database composed of active ROS-1 inhibitors and decoy set molecules.

\begin{tabular}{ccc}
\hline Set No. & Parameters & Values \\
\hline 1 & Total number of compounds in the database (D) & 78 \\
2 & Total number of active compounds in the database (A) & 20 \\
3 & Total number of hits retrieved by pharmacophore model & 25 \\
4 & from the database $(\mathrm{Ht})$ & 20 \\
5 & Total number of active compounds in the hit list $(\mathrm{Ha})$ & 80 \\
6 & \% Yield of active $((\mathrm{Ha} / \mathrm{Ht}) \times 100)$ & 100 \\
7 & \% Ratio of actives $((\mathrm{Ha} / \mathrm{A}) \times 100)$ & 0 \\
8 & False negatives $(\mathrm{A}-\mathrm{Ha})$ & 5 \\
9 & False positives $(\mathrm{Ht}-\mathrm{Ha})$ & 0.77 \\
\hline
\end{tabular}

\subsection{Drug-Like Flavonoids Retrieved by Virtual Screening}

The well-validated Pharmacophore_01 model was used as a 3D query to screen the TimTec database of 4560 flavonoids, mapping a total of 2292 compounds. Subsequently, three filters were applied to the obtained compounds to achieve drug-like flavonoids. A total of 2126 flavonoids passed the Lipinski's rule of five (Ro5) and Veber's filter and this number was further reduced to 19 flavonoids following the absorption, distribution, metabolism, excretion and toxicity (ADMET) pharmacokinetic (PK) properties. These 19 flavonoid compounds were taken forward for the process of molecular docking with ROS-1 kinase domain along with co-crystallized ligand, lorlatinib as a reference compound (Figure 2). The docking scores and interactions of flavonoids with the residues of the ROS-1 kinase domain catalytic pocket (Leu1951, Gly1952, Gly1954, Val1959, Glu1961, Lys1976, Ala1978, Lys1980, Leu2010, Leu2026, Glu2027, Leu2028, Met2029, Glu2030, Gly2032, Asp2033, Glu2061, Arg2083, Asn2084, Leu2086, Gly2101 and Asp2102) were analyzed and compared with that of lorlatinib.

\subsection{Molecular Docking of Retrieved Flavonoids with ROS-1 Tyrosine Kinase Domain}

The drug-like flavonoids derived from the virtual screening of the TimTec database were subjected to molecular docking analysis with the catalytic site of the ROS-1 tyrosine kinase domain. Before docking of flavonoids, the capability of GOLD docking software was assessed by re-docking of the co-crystallized ligand, lorlatinib in the ROS-1 binding pocket. This process resulted in an acceptable root-mean square deviation (RMSD) of $0.83 \AA$ (Figure S1) between the docked pose and available lorlatinib co-crystallized pose. Consequently, the docking of 19 flavonoid molecules was carried out to evaluate their interactions with the aforementioned residues of the ROS- 1 catalytic site. With a Gold score of 43.74 for lorlatinib, a total of 10 flavonoids displayed docking scores higher than the reference compound and were observed to interact with the key residues of the ROS-1 kinase domain (Table S1). More particularly, the interactions of acquired flavonoids with Gly2032 in the WT ROS-1 kinase domain were observed, as characterized by van der Waals interaction. These 10 flavonoids, along with lorlatinib, were also docked with the MT ROS-1 kinase domain, where Gly2032 was mutated to Arg2032. A total of nine flavonoids 
demonstrated higher docking scores and favorable interactions with the mutated kinase domain (Table S2). In particular, the nine compounds formed interactions with Arg2032 via hydrogen or hydrophobic bonds. Furthermore, the binding stability of the identified flavonoids with WT and MT ROS-1 kinase domain was assessed for steric clashes via MD simulations in the next step.

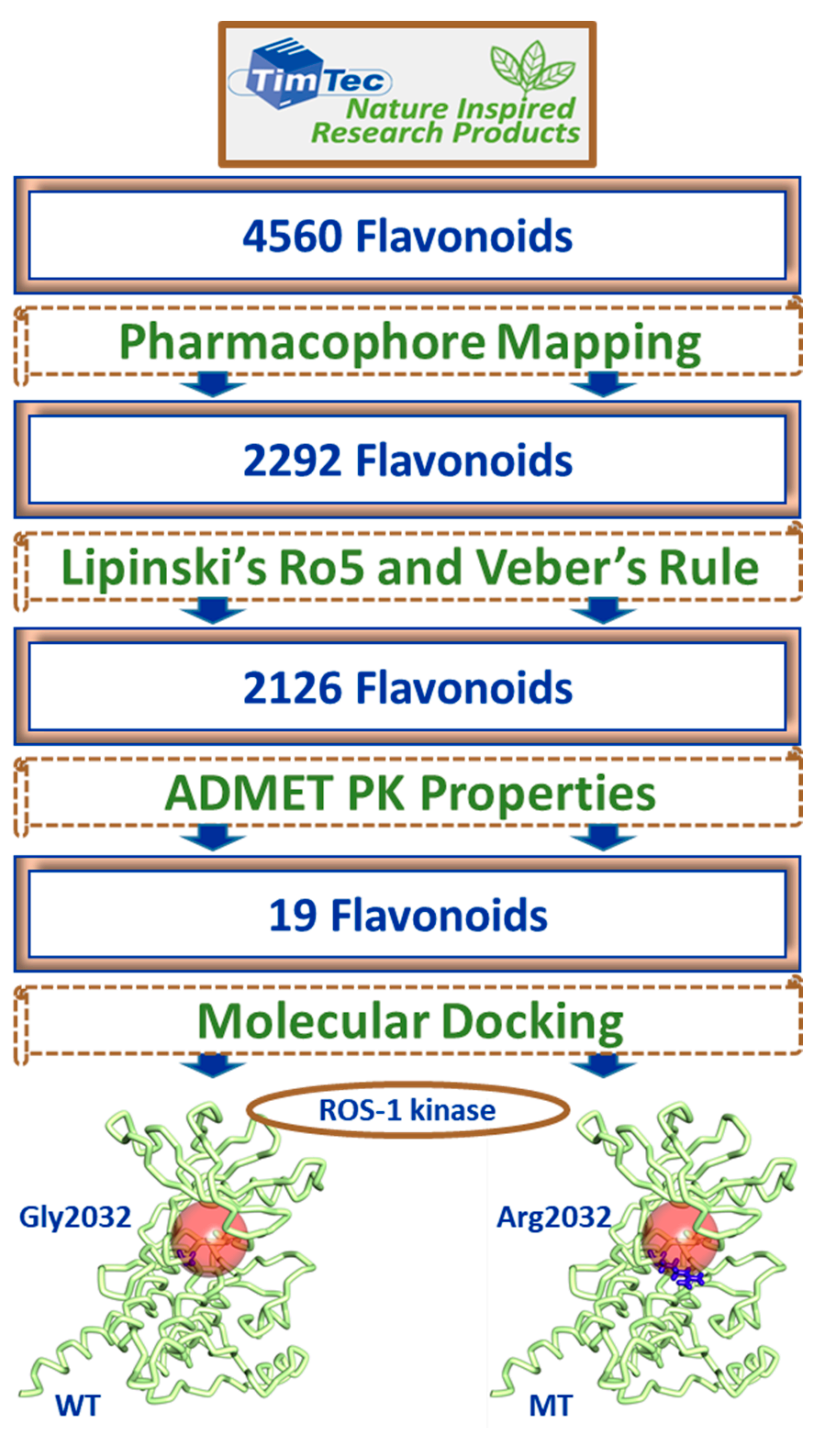

Figure 2. Representation of the steps involved in retrieving drug-like flavonoids for molecular docking, from the TimTec database using the receptor-ligand pharmacophore model.

2.5. Binding Mode, Interaction and Free Energy Analysis of Identified Flavonoids by Molecular Dynamics Simulations

MD simulations were employed for the identified flavonoids and lorlatinib with the WT and MT ROS-1 kinase to check their stabilities for 50 ns. A total of 22 docking systems $(11 \mathrm{WT}+11 \mathrm{MT})$ were taken as initial co-ordinates for MD. The binding stability of the flavonoids was analyzed in terms of their backbone RMSD plots. The RMSD values were observed to be in the range of $0.1-0.4 \mathrm{~nm}$, indicating the stability of the flavonoids with both WT and MT kinase domains (Figure 3). 
(A)

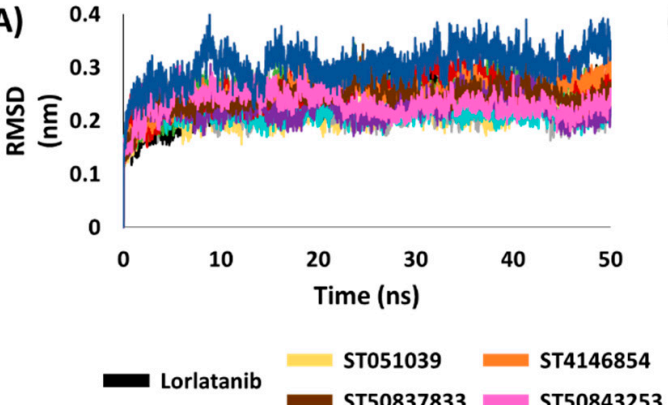

(B)

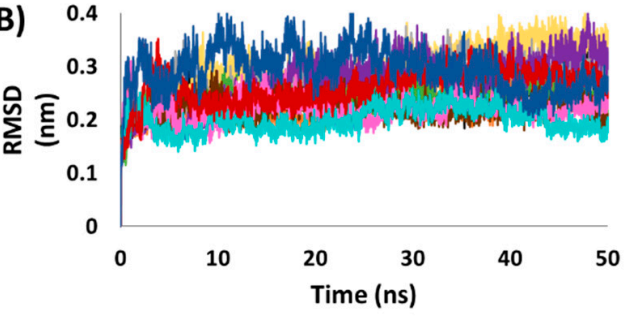

$\begin{array}{lrr}\text { ST052370 } & \text { ST096317 } \\ \text { ST077081 ST50707205 } & \text { ST063011 }\end{array}$

Figure 3. Backbone root-mean-square deviation (RMSD) analysis of (A) wild-type (WT) and (B) mutated (RT) ROS-1 systems.

The representative structures from the last $10 \mathrm{~ns}$ of stable MD trajectories were extracted and superimposed to observe the binding mode of the flavonoids in both the WT and MT ROS-1 kinase catalytic pocket. The flavonoids were perceived to exhibit a similar binding mode as the ROS-1 co-crystallized inhibitor, lorlatinib (Figure 4). The binding interaction of the acquired flavonoids with both the WT and MT ROS- 1 further indicated that the compounds formed requisite bonds with the aforementioned residues of the binding pocket as characterized by hydrogen, van der Waals and hydrophobic interactions (Figures S2 and S3). Furthermore, the compounds did not show steric clashes with the Arg2032 residue of the MT ROS-1 kinase domain.

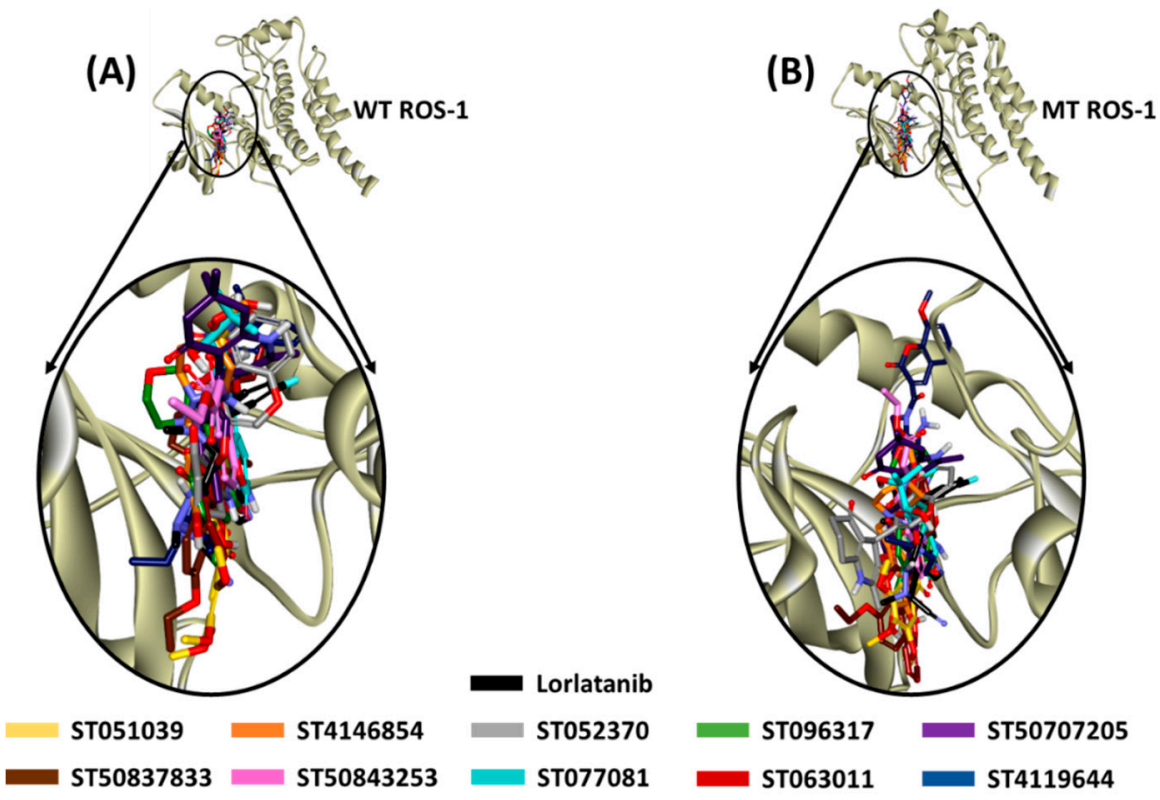

Figure 4. Binding mode of co-crystallized inhibitor, Lorlatinib (reference) and identified flavonoids within the catalytic pocket of (A) WT and (B) MT ROS-1 kinase.

Additionally, the BFE score of the 11 flavonoids with WT and MT ROS-1 was calculated via MM/PBSA and compared with the BFE score of lorlatinib. With the BFE score of $-88.244 \pm 12.933 \mathrm{~kJ} / \mathrm{mol}$ for lorlatinib binding with WT ROS-1, a total of three flavonoids demonstrated better BFE scores (Table S3, Figure S4A). In addition, the BFE score of lorlatinib with MT ROS-1 was computed as $-75.505 \pm 12.053 \mathrm{~kJ} / \mathrm{mol}$, and two flavonoids exhibited superior BFE scores (Table S4, Figure S4B). Only one flavonoid (hereafter referred to as Hit molecule) presented with better BFE scores both with WT $(-91.685 \pm 20.795 \mathrm{~kJ} / \mathrm{mol})$ and MT $(-103.035 \pm 16.223 \mathrm{~kJ} / \mathrm{mol})$ ROS-1 kinase than the aforesaid lorlatinib's BFE with the kinase domains. 
The hydrogen bonds from the equilibrium trajectory of the lorlatinib's interaction with both WT and MT ROS-1 systems were analyzed. Lorlatinib displayed key hydrogen bonding interactions with hinge residues- Glu2027 and Met2029 and also with the solvent front residue Gly2032 (Figure 5, Figure S2 and Table 3). Moreover, no steric clashes with Arg2032 (MT ROS-1) were observed (Figure 6 and Figure S3) as already reported in the literature, thereby explaining the effectivity of lorlatinib in inhibiting crizotinib-resistant NSCLC tumors. Upon scrupulous analysis, it was observed that the Hit molecule with the highest dock score of 72.80 and 73.62 with WT and MT ROS-1 (Tables S1 and S2), respectively maintained its hydrogen bonds with Lys1980 (from the $\beta 3$ sheet) and Met2029 (from the hinge region) as seen in the docking analysis. However, the hydrogen bond with hinge residue Met2029 was weakened during 50 ns and gradually disappeared because of another hydrogen bond with Gly2101 (Hit/WT-ROS-1 interaction) (Figure 5, Figure S2 and Table 3) and Phe2103 (Hit/MT ROS-1 interaction) (Figure 6, Figure S3 and Table 3) from the DFG-motif. From the above meticulous analysis, it was observed that the Hit molecule inserts into DFG-motif, conferring a level of selectivity for ROS-1 over ALK as seen in previous studies by Tian et al. [31,32].

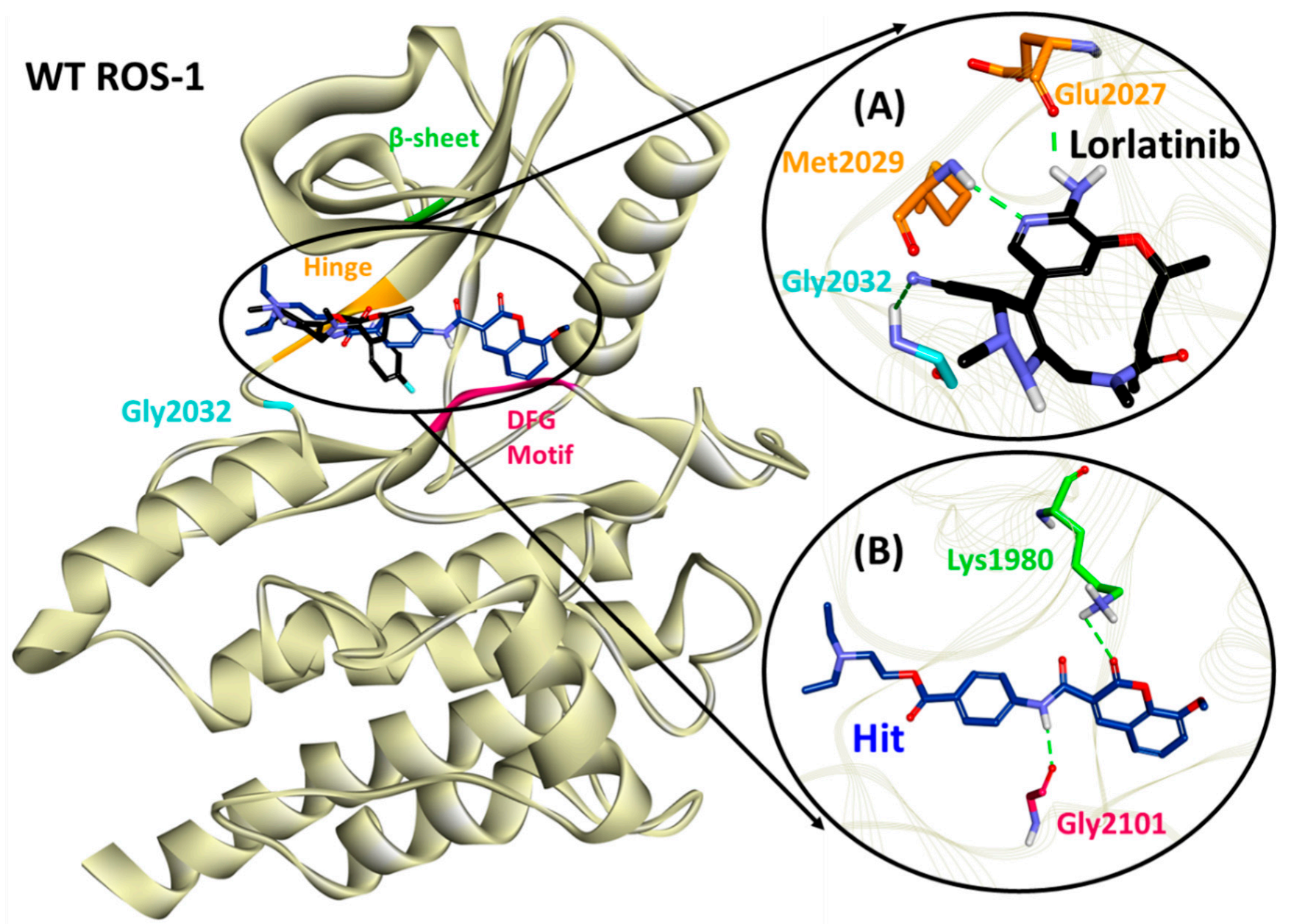

Figure 5. Binding mode of (A) lorlatinib and (B) Hit compound in the wild type (WT) ROS-1 catalytic pocket and molecular interactions with key residues. 


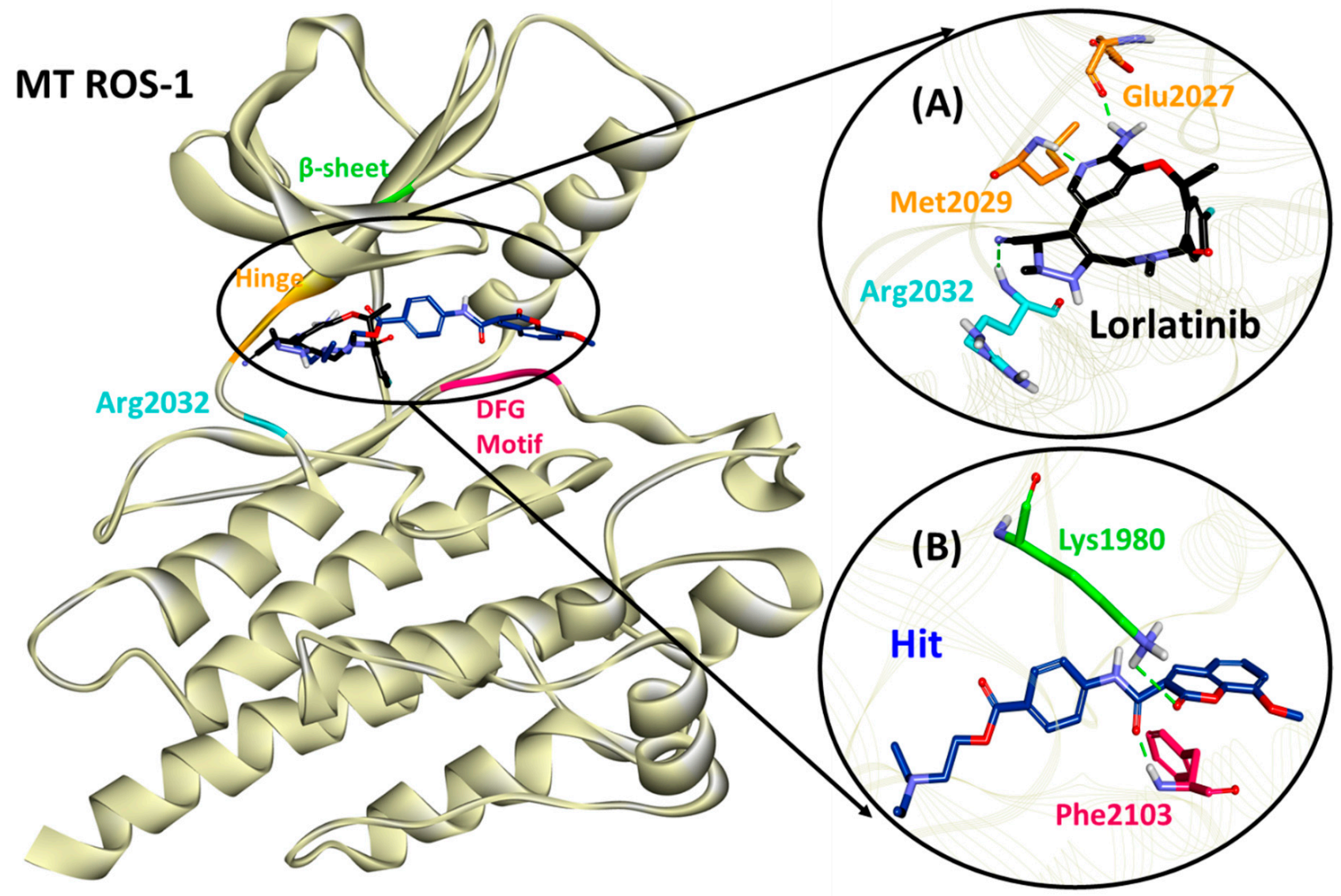

Figure 6. Binding mode of (A) lorlatinib and (B) Hit compound in the mutated (MT) ROS-1 catalytic pocket and molecular interactions with key residues.

Table 3. Molecular interactions between the compounds (lorlatinib and Hit) and the active site residues of wild-type (WT) and mutated (MT) ROS-1 kinase acquired from stable molecular dynamics (MD) trajectories.

\begin{tabular}{|c|c|c|c|c|}
\hline Complex & $\begin{array}{l}\text { Hydrogen Bond } \\
\text { Interactions } \\
\text { (Distance in } \AA \text { ) }\end{array}$ & $\begin{array}{l}\text { Carbon Hydrogen } \\
\text { Bond Interactions }\end{array}$ & $\begin{array}{l}\text { Hydrophobic }(\pi) \\
\text { Interactions }\end{array}$ & $\begin{array}{l}\text { Van der Waals } \\
\text { Interactions }\end{array}$ \\
\hline $\begin{array}{c}\text { Lorlatinib } \\
\text { (with WT ROS-1) }\end{array}$ & $\begin{array}{l}\text { Glu2027 (1.96), } \\
\text { Met2029 (2.21), } \\
\text { Gly2032 (3.05) }\end{array}$ & $\begin{array}{l}\text { Leu1951, Met2029, } \\
\text { Leu2030, Gly2101 }\end{array}$ & $\begin{array}{l}\text { Val1959, Ala1978, Lys1980, } \\
\text { Leu2026, Leu2028 }\end{array}$ & $\begin{array}{l}\text { Gly1952, Leu2010, Leu2028, } \\
\text { Asp2033, Arg2083, Asp2102, }\end{array}$ \\
\hline $\begin{array}{c}\text { Hit } \\
\text { (with WT ROS-1) }\end{array}$ & $\begin{array}{l}\text { Lys1980 (2.81), } \\
\text { Gly2101 (2.03) }\end{array}$ & Met2029 & $\begin{array}{l}\text { Leu1951, Met2001, } \\
\text { Leu2010, Leu2028, } \\
\text { Leu2086, Phe2103 }\end{array}$ & $\begin{array}{c}\text { Ser1953, Glu1961, Glu1997, } \\
\text { Leu2000, Phe2004, Ile2009, } \\
\text { Leu2026, Glu2030, Gly2032, } \\
\text { Asp2102 }\end{array}$ \\
\hline $\begin{array}{c}\text { Lorlatinib } \\
\text { (with MT ROS-1) }\end{array}$ & $\begin{array}{l}\text { Glu2027 (1.81), } \\
\text { Met2029 (1.95), } \\
\text { Arg2032 (2.12) }\end{array}$ & Leu1951, Met2029 & $\begin{array}{l}\text { Val1959, Ala1978, Lys1980, } \\
\text { Leu2026, Leu2086 }\end{array}$ & $\begin{array}{c}\text { Gly1952, Leu2010, Leu2028, } \\
\text { Glu2030, Gly2031, Asp2033, } \\
\text { Asn2084, Asp2102 }\end{array}$ \\
\hline $\begin{array}{c}\text { Hit } \\
\text { (with MT ROS-1) }\end{array}$ & $\begin{array}{l}\text { Lys1980 (2.97), } \\
\text { Phe2103 (1.85) }\end{array}$ & Ala2106 & $\begin{array}{l}\text { Leu1951, Glu1997, } \\
\text { Met2001, Phe2004, } \\
\text { Leu2026, Leu2028, } \\
\text { Arg2032, Phe2103 }\end{array}$ & $\begin{array}{c}\text { Gly1952, Val1959, Ala1978, } \\
\text { Leu2000, Leu2010, Met2029, } \\
\text { Phe2075, Leu2086, Gly2101, } \\
\text { Asp2102, Gly2104 }\end{array}$ \\
\hline
\end{tabular}


As a final assessment, the identified flavonoids were checked whether they were evaluated earlier for ROS-1 inhibitory activity, using the PubChem chemistry database (https:/ / pubchem.ncbi.nlm.nih.gov/, accessed on 5 April 2021). The search results confirmed that the identified flavonoids, including our Hit compound were not tested experimentally for ROS-1 inhibition. The flavonoids also displayed key pharmacophoric features similar to lorlatinib, required for ROS-1 inhibition, as seen from the mapping analysis (Figure 1 and Figure S5). Altogether, these results determine that the acquired flavonoids, especially the Hit compound from the present study, can be recommended as potential inhibitors of ROS-1 kinase.

\section{Discussion}

The orphan RTK c-ros oncogene1 (ROS-1) is a molecular driver in NSCLC patients where approximately 15,000 new patients are estimated to harbor tumors driven by rearranged ROS-1 [14]. Crizotinib was observed to be highly effective against ROS-1 rearranged NSCLC tumors. However, the phylogenic proximity of ROS-1 and ALK led to crizotinib resistance due to the acquisition of Gly2032Arg mutation in the ROS-1 catalytic kinase domain [33]. Lorlatinib was therefore developed for preventing the Arg2032-mediated steric clashes, demonstrating effective activity against both WT ROS- $1\left(\mathrm{IC}_{50}=0.6 \mathrm{nM}\right)$ and MT ROS-1 $\left(\mathrm{IC}_{50}=203 \mathrm{nM}\right)$ with marked selectivity for ROS-1 versus ALK [34,35].

The bioactive polyphenols exhibit therapeutic effects in cancers [36]. These polyphenols constitute flavonoids and non-flavonoids, among which the latter possesses antioxidant, anti-inflammatory and antibacterial activities. In carcinogenesis, flavonoids hinder multiple transduction pathways, limit the proliferation of cancer cells and increase apoptosis [37]. Moreover, they elevate the reactive oxygen species levels [37]. The therapeutic effectivity of flavonoids for the treatment of resistant EGF receptor-mutated NSCLC is discussed in detail [38]. The inhibitory activity of Scutellaria flavonoids was also observed in vitro and in vivo against the overexpression of Id1 in NSCLC [39]. The flavonoid cisplatin also potentiated anti-cancer activity in NSCLC A549 cells in vitro by inhibiting histone deacetylase [40]. The dietary flavonoid, kaempferol was also identified as a potent Nuclear factor erythroid 2-related factor 2 (Nrf2) inhibitor in NSCLC cells using Nrf2 reporter assay [41].

Encouraged from the above-mentioned efforts, we designed a pharmacophore-based virtual screening strategy $[30,35,42-46]$ combined with molecular docking and molecular dynamics simulations to acquire flavonoids as effective WT and MT ROS-1 RTK inhibitors. Accordingly, a receptor-ligand pharmacophore model was developed deriving HBA, HBD and Hy as key pharmacophoric features (Figure 1). The generated pharmacophore model showed a good selectivity score of 7.0747 and was subsequently validated by the decoy set validation method, computing a GF score of 0.77 . The GF score was acquired near the upper limit value of 1 , thereby demonstrating the overall suitability of our model in evaluating active molecules from a given dataset [30,46]. A total of 4560 flavonoids from the TimTec database (https:/ /www.timtec.net/, accessed on 5 April 2021) were screened with our model as query structure, obtaining 2292 flavonoids. Consequently, 19 drug-like flavonoids were retrieved after subjecting the 2292 flavonoids for their drug-likeness properties using Lipinski's Ro5 and ADMET filters (Figure 2). Molecular docking of 19 flavonoids with the WT and MT ROS-1 RTK domain (PDB ID: 4UXL) resulted in obtaining 10 and 9 flavonoids, respectively, with higher docking scores than lorlatinib (Tables S1 and S2). From the docking analysis, the obtained flavonoids demonstrated favorable interactions with the ROS-1 catalytic binding pocket and did not exhibit Arg2032-mediated steric clashes with the MT ROS-1 kinase. The flavonoids were further escalated for molecular dynamics simulations to observe their behavior at physiological conditions and also to gain insight into the key residues required for selective ROS-1 inhibition. The representative structures for the 10 flavonoids were extracted from the stable MD trajectories (Figure 3) and the obtained flavonoids exhibited interactions with the residues of the WT and MT ROS-1 kinase as seen in previous studies [45,47]. Additionally, the flavonoids demonstrated 
no Arg2032-induced steric clashes and instead formed interactions with the residue via hydrophobic and van der Waals bonds (Figures S2 and S3).

Furthermore, the MD simulations were supplemented with MM/PBSA for computing the binding free energies of the respective complexes (Tables S3 and S4). Three (ST4119644, ST50837833 and ST096317) and two (ST4119644 and ST051039) flavonoids presented with better binding energies than lorlatinib with the WT and MT ROS-1 kinase, respectively (Tables S3 and S4). The flavonoid with TimTec ID: ST4119644 was observed as the common entity demonstrating better energy than lorlatinib with both the WT and MT ROS-1 kinase (Figure S4). Therefore, it was considered as a Hit compound. The binding interaction of our Hit in the ROS-1 catalytic pocket was compared with the interaction of lorlatinib. In the case of WT ROS-1, lorlatinib was observed to form hydrogen bonds with residues of the hinge region (Glu2027 and Met2029) and the solvent front (Gly2032) (Figure 5, Table 3), while Hit formed hydrogen bonding interactions with residues of the $\beta$-sheet (Lys1980) and DFGmotif (Gly2101) (Figure 5, Table 3). With MT ROS-1, lorlatinib retained hydrogen bonds with the aforementioned hinge residues and also with the mutated Arg2032 (Figure 6, Table 3), while Hit also retained its hydrogen bonding interaction with Lys1980 and shifted its orientation to form another hydrogen bond with residue Phe2103 of the DFG-motif (Figure 6, Table 3). Recent studies by Tian et al. reported about the catalytic pocket comprising key residues from the P-loop, $\beta$-sheet and the DFG-motif contributing to the high selectivity of compounds with ROS-1 over ALK [31,32]. In addition, Davare et al. also reported about the hydrogen bond with $\beta$-sheet residue Lys1980 in ROS-1/Cabozantinib interaction as acquired from MD simulations, conferring selectivity with ROS-1 [14].

The MM/PBSA calculations facilitate the decomposition of the BFE $\Delta \mathrm{G}_{\text {bind }}$ into identifiable contributions. As observed from the BFE analysis, the van der Waals interactions provided the driving force for the flavonoids binding with WT and MT ROS-1 kinase systems (Tables S3 and S4). Moreover, the contribution of van der Waals interaction in the binding of Hit molecule with both WT and MT ROS-1 kinase was perceived as the highest than lorlatinib and other flavonoids. As described in a previous study, the van der Waals forces dominated the inhibitor binding and also determined the specificity with ROS-1 over ALK [32]. Therefore, from the above analysis, it can be interpreted that our Hit maybe selective for ROS-1 than ALK. Correspondingly, the identified flavonoids including the Hit molecule, also portray the pharmacophoric features required for ROS- 1 inhibition, similar to that of lorlatinib (Figure 1 and Figure S5). Finally, the 2D chemical structures of lorlatinib and the Hit molecule were presented along with their IUPAC names (Figure 7). The in vitro profiling of our identified hit compounds can be performed by cell viability assays and their inhibitory $\mathrm{IC}_{50}$ values against different NSCLC cell lines (HCC78, H3122 and A549) [14] can be calculated. Although the in vitro studies of our identified flavonoid molecules are required to corroborate our findings, the structure-based pharmacophore modeling can be very valuable to design novel compounds as ROS-1 RTK inhibitors in the future. Additionally, our study epitomizes an essential platform for the identification of flavonoids as NSCLC therapeutics. 


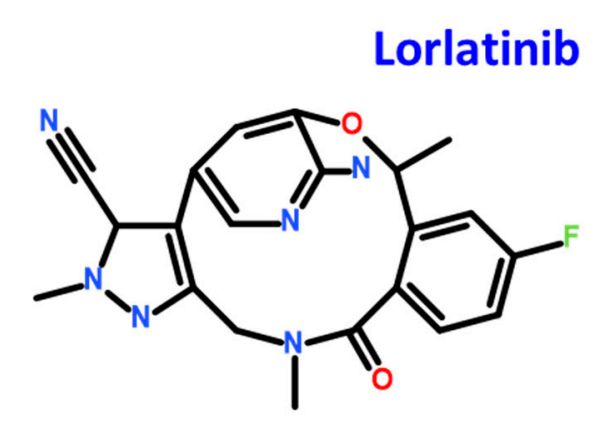

19-Amino-13-fluoro-4,8,16-

trimethyl-9-oxo-17-oxa-4,5,8,20-

tetraazatetracyclo[16.3.1.0 $0^{2,6} \cdot 0^{10,15}$

]docosa-1(22),2,5,10,12,14,18,20-

octaene-3-carbonitrile

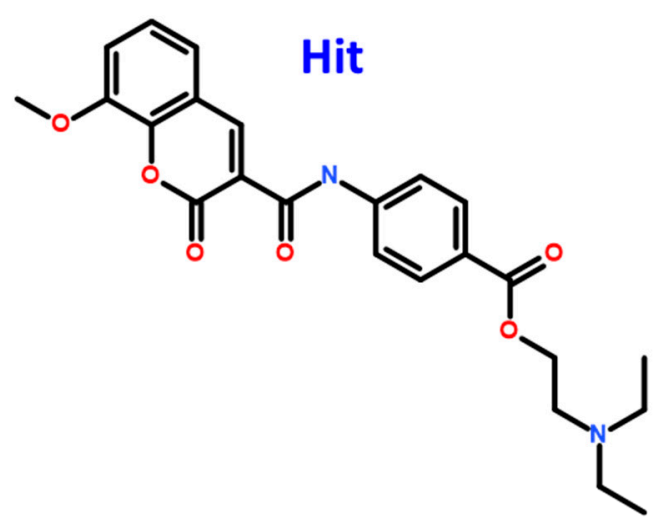

2-(Diethylamino)ethyl 4-\{[(8methoxy-2-oxo-2H-chromen-3yl)carbonyl]amino\}benzoate

Figure 7. The 2D structures of lorlatinib and Hit flavonoid compound of ROS-1 tyrosine kinase.

\section{Materials and Methods}

\subsection{Receptor-Ligand Pharmacophore Model Generation}

The structure-based pharmacophore model explores the catalytic site of the protein interaction with its bound co-crystallized ligand. The information obtained from this pharmacophore model helps in identifying key pharmacophoric features responsible for protein inhibition $[48,49]$. The structure of ROS-1 bound with its co-crystallized inhibitor, Lorlatinib (PDB ID: 4UXL, $2.40 \AA$ ) [16], was obtained from the Protein Data Bank (PDB) and considered for receptor-ligand pharmacophore generation. The Receptor-Ligand Pharmacophore Generation module implanted in Discovery Studio (DS) v.2018 was utilized to generate the model. Given a receptor-ligand interaction, this module works in two steps to create a selective pharmacophore model. In the first step, six pre-defined standard features, including hydrogen bond acceptor (HBA), hydrogen bond donor (HBD), positive ionizable (PI), negative ionizable (NI), ring aromatic (RA) and hydrophobic (Hy), are identified matching the interaction between the protein with its binding ligand [50,51]. The pharmacophore models are ranked in the second step and the model selectivity is estimated based on the genetic function approximation (GFA) model [51]. Subsequently, the model is chosen based on its selectivity score.

\subsection{Validation of Receptor-Ligand Pharmacophore Model}

The validation of the generated pharmacophore model is carried out to assess the pharmacophore's ability to segregate active compounds from inactive ones [51]. The model generated was validated by the Güner-Henry (GH) approach [52], and the goodness of fit (GF) score was subsequently calculated in the range of 0 to 1 , indicating the null and ideal model, respectively [46] according to the following formula:

$$
\mathrm{GF}=\left(\frac{\mathrm{Ha}}{4 \mathrm{HtA}}\right)(3 \mathrm{~A}+\mathrm{Ht}) \times\left\{1-\frac{\mathrm{Ht}-\mathrm{Ha}}{\mathrm{D}-\mathrm{A}}\right\}
$$

where $\mathrm{D}$ represents an external dataset of known active and inactive compounds of the protein while A denotes the active ROS-1 inhibitors, consequently, the Ht represents the hit molecules retrieved from $\mathrm{D}$, and Ha signifies the active hit molecules obtained. The Ligand Pharmacophore Mapping tool complemented with the FAST algorithm embedded in DS was employed for computing the GF score. 


\subsection{Virtual Screening of TimTec Flavonoids Database}

The 3D database of ligands was prepared by downloading and importing the Flavonoids library of molecules from the TimTec database (https://www.timtec.net/, accessed on 5 April 2021) consisting of 4560 compounds. The validated pharmacophore model was used as a 3D query for database screening to identify molecules as potential ROS-1 inhibitors satisfying the pharmacophoric features, utilizing the Ligand Pharmacophore Mapping module in DS. The potential compounds mapping the pharmacophoric features were further subjected to two filtering criteria- Lipinski's rule of five (Ro5) [53] and Veber's rule [54] by employing the Filter by Lipinski and Veber Rules tool in DS. The Ro5 suggests that the drug is absorbed well when the molecule has $\leq 5$ HBD groups, $\leq 10$ HBA groups, $<500$ Da molecular weight and a $\log \mathrm{P}<5$. Veber's rule entails the rotatable bonds of $\leq 10$ for an orally bioavailable compound. Further filtering for pharmacokinetic properties was performed by absorption, distribution, metabolism, excretion and toxicity (ADMET) analysis for the obtained compounds utilizing the ADMET Descriptors module in DS. The ADMET tool is used to predict pharmacokinetic properties for compounds including human intestinal absorption, blood-brain barrier, aqueous solubility and hepatotoxicity. The drug-like compounds obtained from this criteria are escalated for molecular docking with the crystal structure of ROS-1 kinase.

\subsection{Molecular Docking of Drug-Like Compounds with ROS-1 Tyrosine Kinase}

Molecular docking of the drug-like compounds obtained from the above-mentioned filtering process was performed to generate their bioactive binding poses within the catalytic site of WT and MT ROS-1 kinase. The docking process was carried out by employing the genetic optimization for ligand docking (GOLD) v5.2.2 software [55], and hits were chosen based on clustering, Gold scores [56] and interactions with ROS-1. The 3D crystallographic structure retrieved from PDB, complexed with lorlatinib (PDB ID: 4UXL), was prepared with the Clean Protein module in DS. As the crystallographic structure of MT ROS-1 is not available, the Gly2032Arg mutation was modelled using the Build and Edit Protein tool implemented in DS. Prior to docking, the water molecules [57] and the bound ligand were removed, missing residues were supplemented and the protein was minimized. The performance of GOLD software was checked to ensure efficient docking of drug-like compounds by re-docking Lorlatinib into ROS-1 kinase. Subsequently, the Minimize Ligands protocol in DS was utilized to prepare ligands via minimization before docking with ROS-1. The grid co-ordinates were set as $42.32(\mathrm{X}),-19.55(\mathrm{Y})$ and $-4.86(\mathrm{Z})$ with a radius of $7.00 \AA$ for docking of drug-like compounds where lorlatinib was used as a reference. The potential flavonoids obtained from docking were subjected to molecular dynamics (MD) simulations to check their stability at the atomic level.

\subsection{Molecular Dynamics Simulation and Binding Free Energy Calculations}

MD simulation studies of the potential flavonoids obtained from the docking process were carried out for $50 \mathrm{~ns}$ to check their stability in water at the atomistic level in GROningen MAchine for Chemical Simulations (GROMACS) v2018 [58]. The docked complexes of the flavonoids with WT and MT ROS-1 kinase were used as initial coordinates for MD simulations by generating the protein and ligand topologies with CHARMm27 force field [59] and SwissParam [60], respectively. The system was first solvated with a dodecahedron water box using a TIP3P water model and subsequently neutralized by adding the $\mathrm{Na}^{+}$ counter ions. After the steepest descent energy minimization, a two-fold equilibration protocol was executed with NVT (constant number of particles, volume and temperature) and NPT (constant number of particles, pressure and temperature) of 500 ps each. Bond constraints and the geometry of water molecules during the simulation were monitored by the linear constraint solver (LINCS) [61] and SETTLE [62] algorithms, while the long-range electrostatic interactions were computed using the particle mesh Ewald (PME) [63]. The results obtained from MD studies were analyzed in DS as well as in visual molecular dynamics (VMD) [64] software package. The stability of the flavonoid molecules was 
checked by computing their RMSD values over the entire simulation production run, and their binding orientation in the ROS-1 pocket was analyzed. Additionally, the interaction of the hits with WT and MT ROS-1 catalytic residues was examined and compared with the selective inhibitor, lorlatinib. The presence or absence of steric clashes with the Arg2032 residue of the MT ROS-1 kinase was examined and analyzed. The g_mmpbsa tool of GROMACS was utilized to compute the binding free energy scores of the MD-derived complexes [65]. A total of 50 frames were selected evenly from the last $10 \mathrm{~ns}(40-50 \mathrm{~ns})$ of stable MD trajectories and the free energy of binding $\Delta G_{\text {bind }}$ was calculated according to the following equation of molecular mechanics Poisson-Boltzmann surface area (MM/PBSA):

$$
\Delta \mathrm{G}_{\text {bind }}=\mathrm{G}_{\text {complex }}-\left(\mathrm{G}_{\text {protein }}+\mathrm{G}_{\text {ligand }}\right)
$$

\section{Conclusions}

ROS-1 has emerged as a therapeutic target in various malignancies including NSCLC. In the present study, a structure-based pharmacophore model exploiting the crystal structure of ROS-1 with its bound selective inhibitor, lorlatinib, was generated and used for virtual screening of the TimTec flavonoids database. The obtained flavonoids were filtered for their drug-likeness, and a total of 19 flavonoids were subjected to molecular docking both with the WT and MT ROS-1 kinase domain. The flavonoids displayed interactions with key residues of the binding pocket. In addition, no steric clashes were observed with the Arg2032 residue of the MT ROS-1 kinase domain. Subsequently, molecular dynamics simulations combined with binding free energy calculations of flavonoids, with both the WT and MT systems, revealed one flavonoid (Hit) depicting higher energy than lorlatinib. The Hit compound was observed to inhibit ROS-1 by extending into the DFG-motif selectivity pocket than lorlatinib's binding with the hinge region residues. Our results depicted that the interaction of the Hit compound with DFG-motif was responsible for contributing significantly to the binding affinity with ROS-1. Additionally, the identification of flavonoids signifies a quintessential platform for future drug discovery studies against ROS- 1 fusion-positive NSCLC tumors. We, therefore, believe that the scaffolds of our identified flavonoids can act as frameworks for selective drug optimization against ROS-1 kinase.

Supplementary Materials: The following are available online at, Figure S1. Overlay of the docked pose (green) of lorlatinib with its crystal structure conformation (pink) in PDB ID: 4UXL. Figure S2. Binding interaction of flavonoids with key residues of the wild-type (WT) ROS-1 kinase domain. The flavonoids are represented as sticks. Hydrogen bonds are indicated as green dashed lines, hydrophobic interactions are shown as pink and purple spheres and the van der Waals interactions are displayed as light green spheres. Figure S3. Binding interaction of flavonoids with key residues of the mutated (MT) ROS-1 kinase domain. The flavonoids are represented as sticks. Hydrogen bonds are indicated as green dashed lines, hydrophobic interactions are shown as pink, purple and orange spheres and the van der Waals interactions are displayed as light green spheres. Figure S4. Binding free energy (BFE) analysis of (A) wild-type (WT) and (B) mutated (MT) ROS-1 systems. Figure S5. Alignment of the identified flavonoids with the pharmacophoric features. All flavonoids ((B)-(J)) including the (A) Hit compound represent the HBA (hydrogen bond acceptor), Hy (hydrophobic) and HBD (hydrogen bond donor) features of Pharmacophore_01. Table S1. The docking scores and intermolecular interactions of lorlatinib and flavonoids from TimTec database with ROS-1 wild-type (WT) tyrosine kinase domain. Table S2. The docking scores and intermolecular interactions of lorlatinib and flavonoids from TimTec database with ROS-1 mutated (MT) tyrosine kinase domain. Table S3. Binding free energy scores of the identified flavonoids with wild-type (WT) ROS-1 kinase computed through MM/PBSA methodology. Table S4. Binding free energy scores of the identified flavonoids with mutated (MT) ROS-1 kinase computed through MM/PBSA methodology. 
Author Contributions: Conceptualization, S.P.; methodology, S.P.; software, S.P.; validation, S.P.; formal analysis, S.P. and V.K.; investigation, S.P.; resources, K.W.L.; data curation, S.P., V.K. and K.W.L.; writing—original draft preparation, S.P.; writing-review and editing, S.P. and K.W.L.; visualization, S.P.; supervision, K.W.L.; project administration, K.W.L. and J.C.H.; funding acquisition, J.C.H. All authors have read and agreed to the published version of the manuscript.

Funding: This research was supported by Basic Science Research Program through the National Research Foundation of Korea (NRF) funded by the Ministry of Education (2020R1A6A1A03044344).

Data Availability Statement: Data are contained within the article.

Acknowledgments: This research was supported by the Bio and Medical Technology Development Program of the National Research Foundation (NRF) and funded by the Korean government (MSIT) (No. NRF-2018M3A9A70-57263).

Conflicts of Interest: The authors declare no conflict of interest.

Sample Availability: Samples of the compounds are not available from the authors.

\section{Abbreviations}

\begin{tabular}{|c|c|}
\hline ADMET & absorption: distribution, metabolism, excretion, toxicity \\
\hline $\mathrm{AKT}$ & protein kinase B \\
\hline ALK & anaplastic lymphoma kinase \\
\hline BFE & binding free energy \\
\hline DFG & Asp-Phe-Gly \\
\hline EGF & epidermal growth factor \\
\hline FDA & food and drug administration \\
\hline GF & goodness of fit \\
\hline GFA & genetic function approximation \\
\hline $\mathrm{GH}$ & Güner-Henry \\
\hline GOLD & genetic optimization for ligand docking \\
\hline GROMACS & GROningen MAchine for Chemical Simulations \\
\hline HBD & hydrogen bond donor \\
\hline HBA & hydrogen bond acceptor \\
\hline Hy & hydrophobic \\
\hline $\mathrm{IC}_{50}$ & half maximal inhibitory concentration \\
\hline LINCS & LINear Constraint Solver \\
\hline LUAD & lung adenocarcinoma \\
\hline LUSC & lung squamous cell carcinoma \\
\hline MD & molecular dynamics \\
\hline MT & mutant \\
\hline mTOR & mammalian target of rapamycin \\
\hline $\mathrm{MM} / \mathrm{PBSA}$ & molecular mechanics/poisson-boltzmann surface area \\
\hline NI & negative ionizable \\
\hline Nrf2 & nuclear factor erythroid 2-related factor 2 \\
\hline NVT & constant number of particles, volume and temperature \\
\hline NPT & constant number of particles, pressure and temperature \\
\hline NSCLC & non-small cell lung cancer \\
\hline PDB & protein data bank \\
\hline PI & positive ionizable \\
\hline PI3K & phosphoinositide 3-kinase \\
\hline P-loop & phosphate binding loop \\
\hline PME & Particle mesh Ewald \\
\hline RA & ring aromatic \\
\hline RTK & receptor tyrosine kinase \\
\hline ROS-1 & c-ros oncogene 1 \\
\hline Ro5 & rule of five \\
\hline RMSD & root mean square deviation \\
\hline VMD & visual molecular dynamics \\
\hline WT & wild-type \\
\hline
\end{tabular}




\section{References}

1. Molina, J.R.; Yang, P.; Cassivi, S.D.; Schild, S.E.; Adjei, A.A. Non-small cell lung cancer: Epidemiology, risk factors, treatment, and survivorship. Mayo. Clin. Proc. 2008, 83, 584-594. [CrossRef]

2. Rajurkar, S.; Mambetsariev, I.; Pharaon, R.; Leach, B.; Tan, T.; Kulkarni, P.; Salgia, R. Non-Small Cell Lung Cancer from Genomics to Therapeutics: A Framework for Community Practice Integration to Arrive at Personalized Therapy Strategies. J. Clin. Med. 2020, 9, 1870. [CrossRef] [PubMed]

3. Bubendorf, L.; Büttner, R.; Al-Dayel, F.; Dietel, M.; Elmberger, G.; Kerr, K.; López-Ríos, F.; Marchetti, A.; Öz, B.; Pauwels, P.; et al. Testing for ROS1 in non-small cell lung cancer: A review with recommendations. Virchows Arch. 2016, 469, 489-503. [CrossRef]

4. Camp, E.; Anderson, P.J.; Zannettino, A.C.W.; Gronthos, S. Tyrosine kinase receptor c-ros-oncogene 1 mediates TWIST-1 regulation of human mesenchymal stem cell lineage commitment. Bone 2017, 94, 98-107. [CrossRef]

5. Zhu, V.W.; Upadhyay, D.; Schrock, A.B.; Gowen, K.; Ali, S.M.; Ou, S.H.I. TPD52L1-ROS1, a new ROS1 fusion variant in lung adenosquamous cell carcinoma identified by comprehensive genomic profiling. Lung Cancer 2016, 97, 48-50. [CrossRef]

6. Kohno, T.; Nakaoku, T.; Tsuta, K.; Tsuchihara, K.; Matsumoto, S.; Yoh, K.; Goto, K. Beyond ALK-RET, ROS1 and other oncogene fusions in lung cancer. Transl. Lung Cancer Res. 2015, 4, 156-164. [CrossRef]

7. Shan, L.; Lian, F.; Guo, L.; Qiu, T.; Ling, Y.; Ying, J.; Lin, D. Detection of ROS1 gene rearrangement in lung adenocarcinoma: Comparison of IHC, FISH and real-time RT-PCR. PLoS ONE 2015, 10, e0120422. [CrossRef]

8. Bergethon, K.; Shaw, A.T.; Ou, S.H.I.; Katayama, R.; Lovly, C.M.; McDonald, N.T.; Massion, P.P.; Siwak-Tapp, C.; Gonzalez, A.; Fang, R.; et al. ROS1 rearrangements define a unique molecular class of lung cancers. J. Clin. Oncol. 2012, 30, 863-870. [CrossRef] [PubMed]

9. Kazandjian, D.; Blumenthal, G.M.; Luo, L.; He, K.; Fran, I.; Lemery, S.; Pazdur, R. Benefit-Risk Summary of Crizotinib for the Treatment of Patients With ROS1 Alteration-Positive, Metastatic Non-Small Cell Lung Cancer. Oncologist 2016, 21, 974-980. [CrossRef]

10. Patil, T.; Simons, E.; Mushtaq, R.; Pacheco, J.M.; Doebele, R.C.; Bowles, D.W. Targeted therapies for ROS1-rearranged non-small cell lung cancer. Drugs Today. 2019, 55, 641-652. [CrossRef] [PubMed]

11. Elliott, J.; Bai, Z.; Hsieh, S.C.; Kelly, S.E.; Chen, L.; Skidmore, B.; Yousef, S.; Zheng, C.; Stewart, D.J.; Wells, G.A. ALK inhibitors for non-small cell lung cancer: A systematic review and network meta-analysis. PLoS ONE 2020, 15, e0229179. [CrossRef]

12. Sgambato, A.; Casaluce, F.; Maione, P.; Gridelli, C. Targeted therapies in non-small cell lung cancer: A focus on ALK/ROS1 tyrosine kinase inhibitors. Expert Rev. Anticancer Ther. 2018, 18, 71-80. [CrossRef]

13. Davare, M.A.; Saborowski, A.; Eide, C.A.; Tognon, C.; Smith, R.L.; Elferich, J.; Agarwal, A.; Tyner, J.W.; Shinde, U.P.; Lowe, S.W.; et al. Foretinib is a potent inhibitor of oncogenic ROS1 fusion proteins. Proc. Natl. Acad. Sci. USA 2013, 110, 19519-19524. [CrossRef]

14. Davare, M.A.; Vellore, N.A.; Wagner, J.P.; Eide, C.A.; Goodman, J.R.; Drilon, A.; Deininger, M.W.; O’Hare, T.; Druker, B.J. Structural insight into selectivity and resistance profiles of ROS1 tyrosine kinase inhibitors. Proc. Natl. Acad. Sci. USA 2015, 112, E5381-E5390. [CrossRef]

15. Ye, M.; Zhang, X.; Li, N.; Zhang, Y.; Jing, P.; Chang, N.; Wu, J.; Ren, X.; Zhang, J. ALK and ROS1 as targeted therapy paradigms and clinical implications to overcome crizotinib resistance. Oncotarget 2016, 7, 12289-12304. [CrossRef]

16. Zou, H.Y.; Li, Q.; Engstrom, L.D.; West, M.; Appleman, V.; Wong, K.A.; McTigue, M.; Deng, Y.L.; Liu, W.; Brooun, A.; et al. PF06463922 is a potent and selective next-generation ROS1/ALK inhibitor capable of blocking crizotinib-resistant ROS1 mutations. Proc. Natl. Acad. Sci. USA 2015, 112, 3493-3498. [CrossRef] [PubMed]

17. Menichincheri, M.; Ardini, E.; Magnaghi, P.; Avanzi, N.; Banfi, P.; Bossi, R.; Buffa, L.; Canevari, G.; Ceriani, L.; Colombo, M.; et al. Discovery of Entrectinib: A New 3-Aminoindazole As a Potent Anaplastic Lymphoma Kinase (ALK), c-ros Oncogene 1 Kinase (ROS1), and Pan-Tropomyosin Receptor Kinases (Pan-TRKs) inhibitor. J. Med. Chem. 2016, 59, 3392-3408. [CrossRef] [PubMed]

18. Drilon, A.; Somwar, R.; Wagner, J.P.; Vellore, N.A.; Eide, C.A.; Zabriskie, M.S.; Arcila, M.E.; Hechtman, J.F.; Wang, L.; Smith, R.S.; et al. A novel crizotinib-resistant solvent-front mutation responsive to cabozantinib therapy in a patient with ROS1-rearranged lung cancer. Clin. Cancer Res. 2016, 22, 2351-2358. [CrossRef]

19. D'Angelo, A.; Sobhani, N.; Chapman, R.; Bagby, S.; Bortoletti, C.; Traversini, M.; Ferrari, K.; Voltolini, L.; Darlow, J.; Roviello, G. Focus on ros1-positive non-small cell lung cancer (Nsclc): Crizotinib, resistance mechanisms and the newer generation of targeted therapies. Cancers 2020, 12, 3293. [CrossRef]

20. Zanoaga, O.; Braicu, C.; Jurj, A.; Rusu, A.; Buiga, R.; Berindan-Neagoe, I. Progress in research on the role of flavonoids in lung cancer. Int. J. Mol. Sci. 2019, 20, 4291. [CrossRef]

21. Cui, Y.; Morgenstern, H.; Greenland, S.; Tashkin, D.P.; Mao, J.T.; Cai, L.; Cozen, W.; Mack, T.M.; Lu, Q.Y.; Zhang, Z.F. Dietary flavonoid intake and lung cancer-A population-based case-control study. Cancer 2008, 112, 2241-2248. [CrossRef]

22. Le Marchand, L.; Murphy, S.P.; Hankin, J.H.; Wilkens, L.R.; Kolonel, L.N. Intake of Flavonoids and Lung Cancer. JNCI J. Natl. Cancer Inst. 2000, 92, 154-160. [CrossRef] [PubMed]

23. Wu, Z.; Xu, B.; Yu, Z.; He, Q.; Hu, Z.; Zhou, S.; Chen, M.; Zhu, L. Trifolium Flavonoids Overcome Gefitinib Resistance of Non-Small-Cell Lung Cancer Cell by Suppressing ERK and STAT3 Signaling Pathways. Biomed Res. Int. 2020, 1-8. [CrossRef]

24. Han, L.; Fang, S.; Li, G.; Wang, M.; Yu, R. Total flavonoids suppress lung cancer growth via the COX-2-mediated Wnt/ $\beta-$ catenin signaling pathway. Oncol. Lett. 2020, 19, 1824-1830. [CrossRef] [PubMed] 
25. Park, K.I.; Park, H.S.; Kim, M.K.; Hong, G.E.; Nagappan, A.; Lee, H.J.; Yumnam, S.; Lee, W.S.; Won, C.K.; Shin, S.C.; et al. Flavonoids identified from Korean Citrus aurantium L. inhibit Non-Small Cell Lung Cancer growth in vivo and in vitro. J. Funct. Foods 2014, 7, 287-297. [CrossRef]

26. Chen, H.; Yang, J.; Hao, J.; Lv, Y.; Chen, L.; Lin, Q.; Yuan, J.; Yang, X. A Novel Flavonoid Kushenol Z from Sophora flavescens Mediates mTOR Pathway by Inhibiting Phosphodiesterase and Akt Activity to Induce Apoptosis in Non-Small-Cell Lung Cancer Cells. Molecules 2019, 24, 4425. [CrossRef] [PubMed]

27. Rajendran, P.; Maheshwari, U.; Muthukrishnan, A.; Muthuswamy, R.; Anand, K.; Ravindran, B.; Dhanaraj, P.; Balamuralikrishnan, B.; Chang, S.W.; Chung, W.J. Myricetin: Versatile plant based flavonoid for cancer treatment by inducing cell cycle arrest and ROS-reliant mitochondria-facilitated apoptosis in A549 lung cancer cells and in silico prediction. Mol. Cell. Biochem. 2020, 476, 57-68. [CrossRef] [PubMed]

28. Ye, Q.; Liu, K.; Shen, Q.; Li, Q.; Hao, J.; Han, F.; Jiang, R.W. Reversal of multidrug resistance in cancer by multi-functional flavonoids. Front. Oncol. 2019, 9, 487. [CrossRef]

29. Pathak, D.; Chadha, N.; Silakari, O. Identification of non-resistant ROS-1 inhibitors using structure based pharmacophore analysis. J. Mol. Graph. Model. 2016, 70, 85-93. [CrossRef]

30. Rampogu, S.; Park, C.; Ravinder, D.; Son, M.; Baek, A.; Zeb, A.; Bavi, R.; Kumar, R.; Lee, G.; Parate, S.; et al. Pharmacotherapeutics and molecular mechanism of phytochemicals in alleviating hormone-responsive breast cancer. Oxid. Med. Cell. Longev. 2019, 2019, 1-14. [CrossRef]

31. Tian, Y.; Zhang, T.; Long, L.; Li, Z.; Wan, S.; Wang, G.; Yu, Y.; Hou, J.; Wu, X.; Zhang, J. Design, synthesis, biological evaluation and molecular modeling of novel 2-amino-4-(1-phenylethoxy) pyridine derivatives as potential ROS1 inhibitors. Eur. J. Med. Chem. 2018, 143, 182-199. [CrossRef]

32. Tian, Y.; Yu, Y.; Shen, Y.; Wan, H.; Chang, S.; Zhang, T.; Wan, S.; Zhang, J. Molecular Simulation Studies on the Binding Selectivity of Type-I Inhibitors in the Complexes with ROS1 versus ALK. J. Chem. Inf. Model. 2017, 57, 977-987. [CrossRef] [PubMed]

33. Mark, M.; Awad, M.D.; Katayama, R.; McTigue, M.; Liu, W.; Deng, Y.; Brooun, A.; Friboulet, L.; Huang, D.; Matthew, D.; et al. Acquired Resistance to Crizotinib from a Mutation in CD74-ROS1. N. Engl. J. Med. 2013, 368, 1-9. [CrossRef]

34. Basit, S.; Ashraf, Z.; Lee, K.; Latif, M. First macrocyclic 3rd-generation ALK inhibitor for treatment of ALK/ROS1 cancer: Clinical and designing strategy update of lorlatinib. Eur. J. Med. Chem. 2017, 134, 348-356. [CrossRef]

35. Pathak, D.; Choudhary, S.; Singh, P.K.; Singh, M.; Chadha, N.; Silakari, O. Pharmacophore-based designing of putative ROS-1 targeting agents for NSCLC. Mol. Divers. 2020. [CrossRef]

36. Choudhari, A.S.; Mandave, P.C.; Deshpande, M.; Ranjekar, P.; Prakash, O. Phytochemicals in cancer treatment: From preclinical studies to clinical practice. Front. Pharmacol. 2020, 10, 1614. [CrossRef]

37. Abotaleb, M.; Samuel, S.M.; Varghese, E.; Varghese, S.; Kubatka, P.; Liskova, A.; Büsselberg, D. Flavonoids in cancer and apoptosis. Cancers 2019, 11, 28. [CrossRef] [PubMed]

38. Wang, X.; Chen, B.; Xu, D.; Li, Z.; Liu, H.; Huang, Z.; Huang, K.; Lin, X.; Yao, H. Molecular mechanism and pharmacokinetics of flavonoids in the treatment of resistant EGF receptor-mutated non-small-cell lung cancer: A narrative review. Br. J. Pharmacol. 2021, 6, 1388-1406. [CrossRef]

39. Zhao, Z.; Liu, B.; Sun, J.; Lu, L.; Liu, L.; Qiu, J.; Li, Q.; Yan, C.; Jiang, S.; Mohammadtursun, N.; et al. Scutellaria flavonoids effectively inhibit the malignant phenotypes of non-small cell lung cancer in an id1-dependent manner. Int. J. Biol. Sci. 2019, 15, 1500-1513. [CrossRef]

40. Yan, W.; Wu, T.H.Y.; Leung, S.S.Y.; To, K.K.W. Flavonoids potentiated anticancer activity of cisplatin in non-small cell lung cancer cells in vitro by inhibiting histone deacetylases. Life Sci. 2020, 258, 118211. [CrossRef]

41. Fouzder, C.; Mukhuty, A.; Kundu, R. Kaempferol inhibits Nrf2 signalling pathway via downregulation of Nrf2 mRNA and induces apoptosis in NSCLC cells. Arch. Biochem. Biophys. 2021, 697, 108700. [CrossRef]

42. Parate, S.; Kumar, V.; Lee, G.; Rampogu, S.; Hong, J.C.; Lee, K.W. Marine-Derived Natural Products as ATP-Competitive mTOR Kinase Inhibitors for Cancer Therapeutics. Pharmaceuticals 2021, 14, 282. [CrossRef]

43. Weng, C.-W.; Li, J.-H.; Tsai, J.-Y.; Lin, S.-H.; Chang, G.-C.; Liu, C.-C.; Chen, J.J. Pharmacophore-based virtual screening for the identification of the novel Src inhibitor SJG-136 against lung cancer cell growth and motility. Am. J. Cancer Res. 2020, 10, $1668-1690$.

44. Kumar, A.; Rai, S.; Rathi, E.; Agarwal, P.; Kini, S.G. Pharmacophore-guided fragment-based design of novel mammalian target of rapamycin inhibitors: Extra precision docking, fingerprint-based 2D and atom-based 3D-QSAR modelling. J. Biomol. Struct. Dyn. 2020, 39, 1155-1173. [CrossRef] [PubMed]

45. Vanajothi, R.; Vedagiri, H.; Al-Ansari, M.M.; Al-Humaid, L.A.; Kumpati, P. Pharmacophore based virtual screening, molecular docking and molecular dynamic simulation studies for finding ROS1 kinase inhibitors as potential drug molecules. J. Biomol. Struct. Dyn. 2020, 1-15. [CrossRef]

46. Rampogu, S.; Parate, S.; Parameswaran, S.; Park, C.; Baek, A.; Son, M.; Park, Y.; Park, S.J.; Lee, K.W. Natural compounds as potential Hsp90 inhibitors for breast cancer-Pharmacophore guided molecular modelling studies. Comput. Biol. Chem. 2019, 83, 1-12. [CrossRef]

47. Luo, L.X.; Fan, X.X.; Li, Y.; Peng, X.; Ji, Y.C.; Hsiao, W.W.L.; Liu, L.; Leung, E.L.H.; Yao, X.J. Identification of mitoxantrone as a new inhibitor of ROS1 fusion protein in non-small cell lung cancer cells. Medchemcomm 2017, 8, 621-624. [CrossRef]

48. Salam, N.K.; Nuti, R.; Sherman, W. Novel method for generating structure-based pharmacophores using energetic analysis. J. Chem. Inf. Model. 2009, 49, 2356-2368. [CrossRef] 
49. Meslamani, J.; Li, J.; Sutter, J.; Stevens, A.; Bertrand, H.O.; Rognan, D. Protein-ligand-based pharmacophores: Generation and utility assessment in computational ligand profiling. J. Chem. Inf. Model. 2012, 52, 943-955. [CrossRef]

50. Sutter, J.; Li, J.; J. Maynard, A.; Goupil, A.; Luu, T.; Nadassy, K. New Features that Improve the Pharmacophore Tools from Accelrys. Curr. Comput. Aided-Drug Des. 2011, 7, 173-180. [CrossRef]

51. Khedkar, S.; Malde, A.; Coutinho, E.; Srivastava, S. Pharmacophore Modeling in Drug Discovery and Development: An Overview. Med. Chem. (Los Angeles) 2007, 3, 187-197. [CrossRef]

52. Lin, S.-K. Pharmacophore Perception, Development and Use in Drug Design. Edited by Osman, F. Güner. Molecules 2000, 5, 987-989. [CrossRef]

53. Lipinski, C.A. Lead- and drug-like compounds: The rule-of-five revolution. Drug Discov. Today Technol. 2004, 1, 337-341. [CrossRef]

54. Veber, D.F.; Johnson, S.R.; Cheng, H.Y.; Smith, B.R.; Ward, K.W.; Kopple, K.D. Molecular properties that influence the oral bioavailability of drug candidates. J. Med. Chem. 2002, 45, 2615-2623. [CrossRef] [PubMed]

55. Jones, G.; Willett, P.; Glen, R.C.; Leach, A.R.; Taylor, R. Development and validation of a genetic algorithm for flexible docking. J. Mol. Biol. 1997, 267, 727-748. [CrossRef] [PubMed]

56. Verdonk, M.L.; Cole, J.C.; Hartshorn, M.J.; Murray, C.W.; Taylor, R.D. Improved protein-ligand docking using GOLD. Proteins Struct. Funct. Genet. 2003, 52, 609-623. [CrossRef] [PubMed]

57. Kumar, R.; Parameswaran, S.; Bavi, R.; Baek, A.; Son, M.; Rampogu, S.; Park, C.; Lee, G.; Zeb, A.; Parate, S.; et al. Investigation of novel chemical scaffolds targeting prolyl oligopeptidase for neurological therapeutics. J. Mol. Graph. Model. 2019, 88, 92-103. [CrossRef] [PubMed]

58. Abraham, M.J.; Murtola, T.; Schulz, R.; Páll, S.; Smith, J.C.; Hess, B.; Lindah, E. Gromacs: High performance molecular simulations through multi-level parallelism from laptops to supercomputers. SoftwareX 2015, 1-2, 19-25. [CrossRef]

59. Zhu, X.; Lopes, P.E.M.; MacKerell, A.D. Recent developments and applications of the CHARMM force fields. WIREs Comput. Mol. Sci. 2012, 2, 167-185. [CrossRef]

60. Zoete, V.; Cuendet, M.A.; Grosdidier, A.; Michielin, O. SwissParam: A fast force field generation tool for small organic molecules. J. Comput. Chem. 2011, 32, 2359-2368. [CrossRef]

61. Hess, B.; Bekker, H.; Berendsen, H.J.C.; Fraaije, J.G.E.M. LINCS: A Linear Constraint Solver for molecular simulations. J. Comput. Chem. 1997, 18, 1463-1472. [CrossRef]

62. Miyamoto, S.; Kollman, P.A. Settle: An analytical version of the SHAKE and RATTLE algorithm for rigid water models. J. Comput. Chem. 1992, 13, 952-962. [CrossRef]

63. Darden, T.; York, D.; Pedersen, L. Particle mesh Ewald: An N. $\log (\mathrm{N})$ method for Ewald sums in large systems. J. Chem. Phys. 1993, 98, 10089-10092. [CrossRef]

64. Humphrey, W.; Dalke, A.; Schulten, K. VMD: Visual molecular dynamics. J. Mol. Graph. 1996, 14, 33-38. [CrossRef]

65. Kumari, R.; Kumar, R.; Lynn, A. G-mmpbsa -A GROMACS tool for high-throughput MM-PBSA calculations. J. Chem. Inf. Model. 2014, 54, 1951-1962. [CrossRef] [PubMed] 